\title{
Open Access Open Access Scientific Reports

\section{Development of a Novel LC/MS/MS Extraction Assay for Galanthamine in Guinea Pig Plasma and its Application to Nerve Agent Countermeasures}

Wes E. Steiner ${ }^{1 *}$, loannis A. Pikalov ${ }^{1}$, Patrick T. Williams ${ }^{2}$, William A. English² and Corey J. Hilmas ${ }^{3}$

${ }^{1}$ Department of Chemistry and Biochemistry, Eastern Washington University, 226 Science Building, Cheney, WA 99004, USA

${ }^{2}$ Neurobehavioral Toxicology Branch, Analytical Toxicology Division, U.S. Army Medical Research Institute of Chemical Defense, 3100 Ricketts Point Road, Aberdeen Proving Grounds, MD 21010, USA

${ }^{3}$ Division of Dietary Supplement Programs, Office of Nutrition, Labeling, and Dietary Supplements, Center for Food Safety and Applied Nutrition, U.S. Food and Drug Administration, 5100 Paint Branch Parkway, College Park, MD 20740, USA

\begin{abstract}
Galanthamine Hydrobromide (GAL HBr), approved material for treatment of mild to moderate Alzheimer's disease, is a centrally-acting reversible Acetylcholinesterase Inhibitor (AChEI) that is currently under evaluation as a therapeutic countermeasure against organophosphorus G- and V-Series nerve agents, which can induce rapid lethality in guinea pigs and humans. It has been shown that upon combination with Atropine (ATR) and pyridine-2Aldoxime Methochloride (2-PAM), a single dose of GAL administered before or soon after the acute exposure to a lethal dose of organophosphorus compounds can safely counteract toxicity in guinea pigs. To that end a new sample preparation extraction method analysis assay has been developed to enable future high-throughput, reproducible, and sensitive assays to quantitate galanthamine in guinea pig plasma. Samples were prepared with Diphenhydramine Hydrochloride $(\mathrm{DPH} \mathrm{HCl})$ internal standard and recovered with a 10 min liquid-liquid trichloromethane extraction. Samples were analyzed with a reversed phase liquid chromatographic column interfaced to a triple quadrupole mass spectrometer (LC/MS/MS) operating in the positive ion Multiple Reaction Monitoring (MRM) Turbo lonspray mode. Precursor to product ion $(\mathrm{M}+\mathrm{H})+$ transitions of $288-$ to- $213 \mathrm{~m} / \mathrm{z}$ and $256-$ to- $167 \mathrm{~m} / \mathrm{z}$ for GAL and DPH were observed, respectively. Sample run times of $1.50 \mathrm{~min}$ were achieved. Overall extraction method development proved to be acceptable and rugged.
\end{abstract}

Keywords: Guinea Pig; Galanthamine; Liquid chromatography; Mass spectrometry; Chemical warfare agents; Nerve agents

\section{Introduction}

Organophosphorus compounds, since their early introduction in agriculture as a pesticide and later as Weapons of Mass Destruction (WMD) in the form of Chemical Warfare Agents (CWA), are a global concern for their toxicity in humans [1-3]. Organophosphorus G-Series (Sarin (GB), Cyclosarin (GF), Soman (GD), Tabun (GA)), and V-Series (VE, VG, VM, VX) compounds are one classification of CWA by convention, which act to severely disrupt neurological regulation within biological systems through their irreversible inhibition of the enzyme Acetylcholinesterase (AChE) [4-6]. The degree of toxicity (e.g. chronic to acute) or severity of the disruption (e.g. mild to severe) arises from this direct inhibition of the AChE enzyme which causes the excessive accumulation of the neurotransmitter Acetylcholine (ACh) to build up in synaptic junctions. This excessive accumulation of ACh in turn causes systemic symptomatic overstimulation of Ach receptors. The subsequent cholinergic crisis is indicative of muscarinic receptor overstimulation (miosis, lacrimation, bradycardia, bronchoconstriction, hypotension, and diarrhea), nicotinic receptor overstimulation and desensitization (intense skeletal muscle fasciculations followed by subsequent muscle weakness), and central nervous system related effects (nystagmus, ocular flutter, anxiety, restlessness, confusion, ataxia, tremors, seizures, respiratory paralysis, coma, and death) [7-10].

Currently, the standard approved therapeutic treatments to decrease slow aging organophosphorus agent toxicity like VX agents include Atropine sulfate (ATR) to antagonize the muscarinic response, Pyridine-2-Aldoxime Methochloride (2-PAM) to reactivate inhibited AChE enzymes, and Benzodiazepines (BZD) to mitigate convulsant seizures [9-11]. For rapidly aging organophosphorus agents such as soman, pretreatment prophylaxis with the carbamate Pyridostigmine
Bromide (PB) is necessary before post-exposure treatment with ATR, 2-PAM, and BZD [12-14]. All of these treatments; however, have significant well recognized limitations [15-17]. Galanthamine Hydrobromide (GAL HBr), on the other hand, is an alkaloid synthesized synthetically or as an extract from the bulb and/or flowers of the Galanthus Caucasicus plant. GAL has been approved for the treatment of mild to moderate Alzheimer's disease and has been sold as a dietary supplement for memory support. GAL has properties that make it a suitable candidate as a prophylactic and therapeutic countermeasure against organophosphorus poisoning. GAL is a centrally acting reversible Acetylcholinesterase Inhibitor (AChEI) that can cross the blood-brain barrier, exhibit anticonvulsant properties, and prevent neurodegeneration [18-21]. Thus, GAL is currently being studied by several investigators as a countermeasure against organophosphorus nerve agent-induced lethality in rodent animal models [22-24]. When administered alone, ATR and 2-PAM do not enhance survival against lethal nerve agent challenge in guinea pigs. However, when combined with ATR and 2-PAM, a single dose of GAL administered before or soon after the acute exposure to a lethal dose of slow or rapid aging organophosphorus nerve agent compounds GAL can safely counteract their toxicity in guinea pigs [23].

${ }^{*}$ Corresponding author: Wes E. Steiner, Department of Chemistry and Biochemistry Eastern Washington University, 226 Science Building, Cheney, WA 99004, USA, Tel: (509) 359-6521; Fax: (509) 359-6973; E-mail: wsteiner@ewu.edu

Received March 06, 2012; Published July 25, 2012

Citation: Steiner WE, Pikalov IA, Williams PT, English WA, Hilmas CJ (2012) Development of a Novel LC/MS/MS Extraction Assay for Galanthamine in Guinea Pig Plasma and its Application to Nerve Agent Countermeasures. 1: 149. doi:10.4172/ scientificreports. 149

Copyright: (c) 2012 Steiner WE, et al. This is an open-access article distributed under the terms of the Creative Commons Attribution License, which permits unrestricted use, distribution, and reproduction in any medium, provided the original author and source are credited. 
In contrast to other rodent models, guinea pigs, have lower levels of circulating Butyryl Cholinesterase (BuChE) enzymes (e.g. scavengers for CWAs) in their blood stream similar to nonhuman primates and humans, which in turn makes guinea pigs an appropriate nonprimate animal model to predict the effectiveness of GAL therapy for organophosphorus poisoning in humans $[17,22,25,26]$. To this end, it is imperative to accurately and precisely quantitate GAL in guinea pig plasma. Interestingly enough only a few assays have been reported for the quantitation of GAL in biological plasma fluids. The first development was in the use of high performance Liquid Chromatography (LC) combined with ultra-violet or fluorescence detection [27-29]. These assays all suffered from high plasma sample volume requirements, extended time consuming extraction procedures, low sensitivity, and low selectivity. Realizing these sample preparation and instrumental shortcomings researchers soon moved on to techniques that not only employed the separation power of LC but combined that with the low plasma sample requirement, high sensitivity, and high selectivity of triple quadrupole Mass Spectrometry (MS/MS) detection [30-32]. While these assays have shown a variety of improvements they have all been primarily developed for human plasma samples and have not been developed for the rapid extraction and quantitation of therapeutic doses of GAL in guinea pig plasma. With this in mind this paper seeks to expand upon the use of LC/MS/MS to explore the feasibility of using a new sample preparation extraction method analysis assay with Diphenhydramine Hydrochloride ( $\mathrm{DPH} \mathrm{HCl}$ ) as an internal standard to enable a high-throughput, reproducible, and sensitive assay for the extraction of galanthamine in guinea pig plasma.

\section{Experimental Methods}

\section{Chemicals and reagents}

GAL $\mathrm{HBr}$ (purity $\geq 99 \%$ by HPLC) and DPH $\mathrm{HCl}$ (purity $\geq 99 \%$ by HPLC) used as standard reference material and internal standard reference material was purchased from Tocris Biosciences (Ellisville, $\mathrm{MO}$ ). The chemical structures of GAL $\mathrm{HBr}$ and $\mathrm{DPH} \mathrm{HCl}$ are shown in Figure 1. Organophoshorus nerve agent free guinea pig plasma, containing sodium heparin as an anticoagulant, was obtained from Valley Biomedical (Winchester, VA) and the United States Army Medical Research Institute of Chemical Defense (APG, MD). HPLC grade methanol and acetonitrile were obtained from Fisher Scientific (Pittsburgh, PA). Formic acid, glacial acetic acid, hexane, carbon tetrachloride, toluene, t-butyl methyl ether, methylene chloride, and trichloromethane were purchased from Sigma Aldrich (St. Louis, MO). HPLC grade 18.0 M $\Omega$ water was provided by an in house E-Pure water system from Barnstead (Rockford, IL).

\section{Standards and quality controls}

Stock solutions of the GAL standard reference material and the DPH internal standard reference material was prepared separately in methanol at concentrations of $1.0 \mathrm{mg} / \mathrm{mL}$. Serial dilutions of the GAL and DPH stock solutions were made with a water methanol (50:50, $\mathrm{v} / \mathrm{v}$ ) solution to obtain working solutions of $0.05-12.5 \mu \mathrm{g} / \mathrm{mL}$ and 1.25 $\mu \mathrm{g} / \mathrm{mL}$ respectively. Stock and working solutions were stored at $-80^{\circ} \mathrm{C}$ until needed.

\section{Guinea pig plasma samples}

All $160 \mu \mathrm{L}$ guinea pig plasma samples were vortex mixed for 10 s with spiked $40 \mu \mathrm{L}$ aliquots of $1.25 \mu \mathrm{g} / \mathrm{mL}$ DPH internal standard working solution. GAL extraction was then accomplished by the addition of $1.4 \mathrm{~mL}$ of trichloromethane followed by 2 min of vortex mixing and $3 \mathrm{~min}$ of centrifugation at $2000 \mathrm{~g}$. The upper organic layer
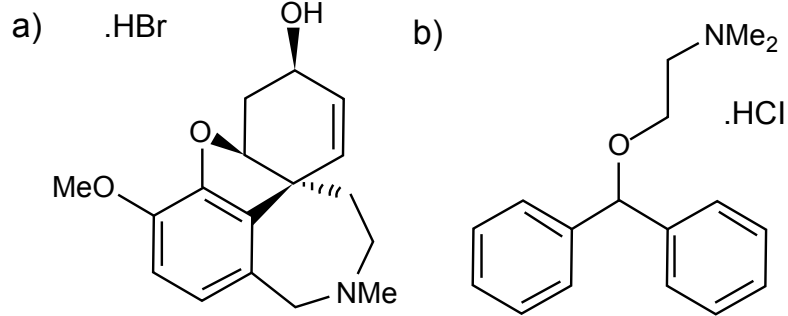

Figure 1: Chemical structures of a) Galanthamine hydrobromide (GAL HBr) and $\mathrm{b}$ ) diphenhydramine hydrochloride ( $\mathrm{DPH} \mathrm{HCl}$ ) internal standard.

was evaporated to dryness under nitrogen at $45^{\circ} \mathrm{C}$ for $5 \mathrm{~min}$ and the dry remaining residue was reconstitution with $100 \mu \mathrm{L}$ of HPLC mobile phase $(35 \% \mathrm{~B})$.

\section{Mass spectrometry instrumentation}

The mass spectrometers systems that were used in this study were a Shimadzu LCMS-2010EV series single quadrupole mass spectrometer (Pleasanton, CA) for repetitive daily assay iterations and a Thermo TSQ Vantage (West Palm Beach, FL) and AB-SCIEX API 4000 (Foster City, CA) series triple quadrupole mass spectrometers for exacting assay development. A typical injection volume of $10 \mu \mathrm{L}$ were made with an Agilent 1100 series HPLC system (Santa Clara, CA) that was pumped at a flow rate of $800 \mu \mathrm{L} / \mathrm{min}$ with a continuous isocratic ratio of $(65: 35, \mathrm{v} / \mathrm{v})$ mobile phase A $(0.05 \%$ formic acid, $0.2 \%$ glacial acetic acid, $99.75 \%$ HPLC grade water) to mobile phase B $(0.1 \%$ formic Acid, 9.9\% HPLC grade water, $90 \%$ methanol). The guard (3.9 $\mathrm{mm} \mathrm{x}$ $20 \mathrm{~mm}$ ) and analytical (4.6 $\mathrm{mm} \times 75 \mathrm{~mm}$ ) columns were both Waters Symmetry Shield $3.5 \mu \mathrm{m}$ packed RP18 columns (Milford, MA) held isothermally at $28^{\circ} \mathrm{C}$. Chromatographic run times for each injection was 1.50 min with retention times of 0.83 and 1.17 min being found for GAL and DPH, respectively. The analytical column eluent was then introduced into the AB-SCIEX API 4000 series triple quadrupole mass spectrometer, which was operating in the positive ion Multiple Reaction Monitoring (MRM) Turbo IonSpray mode. The main source dependent parameters (15 psi ion source gas 1 (GS1): 5 psi ion source gas 2 (GS2); $350^{\circ} \mathrm{C}$ source gas temperature (TEM); 15 psi curtain gas (CUR); $2700 \mathrm{~V}$ ion spray voltage (IS); on was the interface heater (IH)), compound dependent parameters (70 V declustering potential (DP); $10 \mathrm{~V}$ entrance potential (EP); $25 \mathrm{~V}$ collision energy (CE); 7 psi collision gas (CAD); $15 \mathrm{~V}$ collision cell exit potential (CXP); $200 \mathrm{~ms}$ dwell time (DT)), and detector dependent parameters (1730 V detector voltage (CEM)) were all found to be optimal for precursor to product ion $(\mathrm{M}+\mathrm{H})+$ transitions of 288 -to- $213 \mathrm{~m} / \mathrm{z}$ and 256 -to- $167 \mathrm{~m} / \mathrm{z}$ for GAL and $\mathrm{DPH}$, respectively. Data acquisition and processing was performed with AB-SCIEX Analyst Software version 1.4.2 (Foster City, CA) running on a standard Dell Precision 390 personnel computer (PC) system (St. Louis, MO).

\section{Results and Discussion}

\section{Sample preparation development}

Organic solvents (hexane, carbon tetrachloride, toluene, methyl t-butyl ether, methylene chloride, and trichloromethane) were selected as potential candidates via their type, Snyder polarity index (P'), and boiling point to maximize GAL and DPH liquid-liquid extraction recovery from guinea pig plasma [33]. Depending upon which type of organic extraction solvent was used the P' for that solvent consisted of three main contributions, xe (interaction with ethanol - proton 


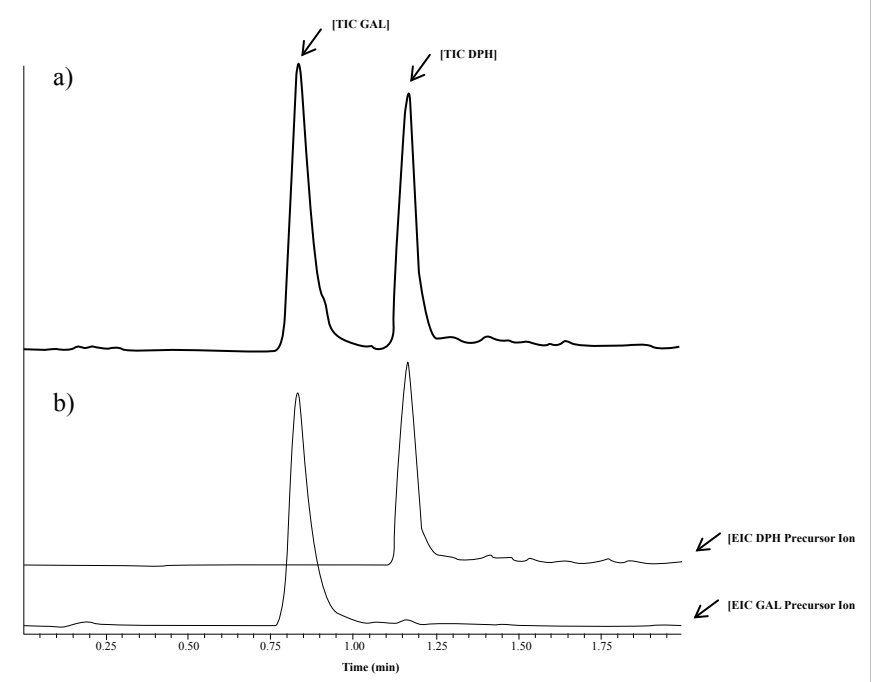

Figure 2: Positive ion mode chromatograms with a) being the total precursor ion current (TIC) chromatogram of a mixture of GAL and DPH and b) the extracted precursor ion current (EIC) chromatograms for GAL and DPH with retention times being found to be 0.83 and 1.17 min, respectively.

acceptors), $x d$ (interaction with dioxane - proton donors) and $x n$ (interaction with nitromethane - dipole-dipole interactions) that could be fine tuned for chromatographic separation integrity and extraction recovery selectivity. Consideration was also given in the selection process to organic solvents with lower boiling points in an effort to increase the rate at which the upper organic layer could be evaporated in the sample preparation process. Thereby, minimizing the rate-limiting time step needed for sample preparation. Ultimately, trichloromethane with a $\mathrm{P}^{\prime}$ value of 4.1 (e.g. 0.1 for non-polar hexane and 9.0 for polar water) and a boiling point of around $61^{\circ} \mathrm{C}$ was used. Optimal extraction recoveries as determined by comparing the peak area for plasma samples for three replicate inter-batch assays that were spiked before and after extraction were obtained for both GAL $(0.025$ (8.69 nM), $0.25(86.9 \mathrm{nM})$, and $1.00 \mu \mathrm{g} / \mathrm{mL}(348 \mathrm{nM}))$ and DPH $(0.25$ $\mu \mathrm{g} / \mathrm{mL}(97.9 \mathrm{nM}))$ in $10 \mathrm{~min}$ with a trichloromethane liquid-liquid extraction.

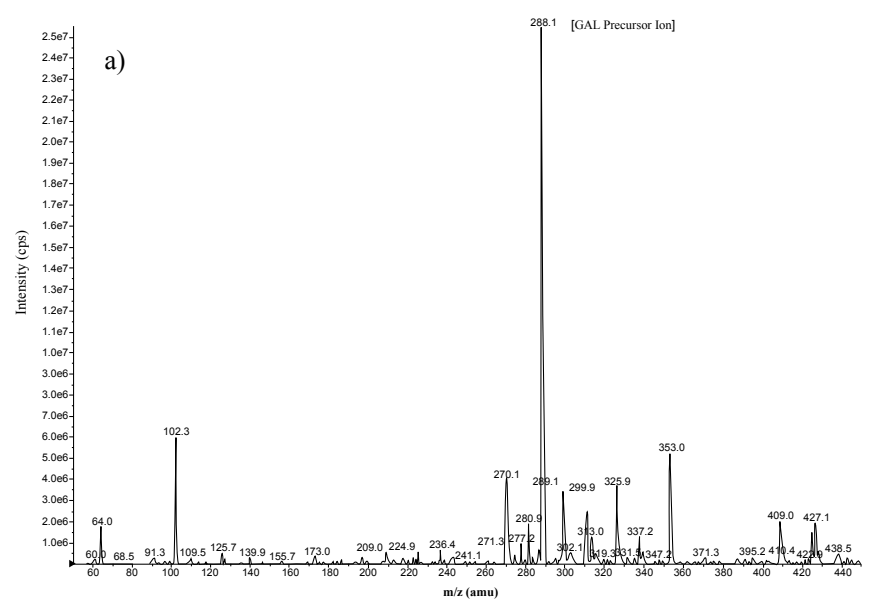

\section{Mass spectrometry development}

Chromatographic mobile phase composition with a continuous isocratic ratio of $(65: 35 \mathrm{v} / \mathrm{v})$ mobile phase A $(0.05 \%$ formic acid, $0.2 \%$ glacial acetic acid, $99.75 \%$ HPLC grade water) to mobile phase B $(0.1 \%$ formic acid, 9.9\% HPLC grade water, 90\% methanol) was found to be optimal for both chromatography and mass spectrometry ionization efficiency after many trials when combined with a Waters Symmetry Shield $3.5 \mu \mathrm{m}$ packed RP18 guard $(3.9 \mathrm{~mm} \times 20 \mathrm{~mm})$ and analytical $(4.6$ $\mathrm{mm} \times 75 \mathrm{~mm}$ ) column assembly. From a chromatography perspective the combination of select mobile phase solvent constituents (e.g. methanol organic modifier and formic acid to displace ion pairs) and stationary phase media type (e.g. shielded silanol non-polar RP18 packing) provided an environment in which equilibrium chemistry could take place between the two phases to provide improved Gaussian peak shapes with consistent baseline resolution, as seen in Figure 2, for GAL and DPH in a relatively short amount of time (i.e. 0.83 and $1.17 \mathrm{~min}$, respectively). From a mass spectrometry perspective the use of key mobile phase solvent constituents (e.g. formic and acetic acid) helped to greatly improve the sensitivity of the analysis by increasing the positive ionization efficiency in the source region of the mass spectrometer.

Mass spectrometric positive ion Turbo IonSpray mode quantifier precursor to product ion $(\mathrm{M}+\mathrm{H})+$ transitions of 288 -to- $213 \mathrm{~m} / \mathrm{z}$ and 256-to- $167 \mathrm{~m} / \mathrm{z}$ were found after a few trials to be the most sensitive and selective for GAL and DPH respectively, in this study. Here, the precursor ions (e.g. 288 and 256, GAL and DPH) were selected in Quadrupole 1 (Q1), fragmented by collisionally induced dissociation (CID) in Quadrupole 2 (Q2), and then product ions (e.g. 213 and 167, GAL and DPH) were selected in Quadrupole $3(\mathrm{Q} 3)$. The quantifier precursor to product ion $(\mathrm{M}+\mathrm{H})+$ transition of 288 -to- $213 \mathrm{~m} / \mathrm{z}$ for GAL is shown in Figure 3 as a) full scan Q1 showing the GAL precursor 288 $\mathrm{m} / \mathrm{z}$ ion and $\mathrm{b}$ ) full scan $\mathrm{Q} 3$ showing the GAL product $213 \mathrm{~m} / \mathrm{z}$ ion. In Figure 4, the quantifier precursor to product ion $(\mathrm{M}+\mathrm{H})+$ transition of 256-to- $167 \mathrm{~m} / \mathrm{z}$ for DPH is shown as a) full scan Q1 showing the DPH precursor $256 \mathrm{~m} / \mathrm{z}$ ion and b) full scan Q3 showing the DPH product $167 \mathrm{~m} / \mathrm{z}$ ion. The same type of mass spectrometry ion selection was also done for the qualifier precursor to product ion $(\mathrm{M}+\mathrm{H})+$ transitions of 288 -to- $231 \mathrm{~m} / \mathrm{z}$ and 256 -to- $152 \mathrm{~m} / \mathrm{z}$ for GAL and DPH, respectively. This ensured that if a case did happen to arise in which the quantifier

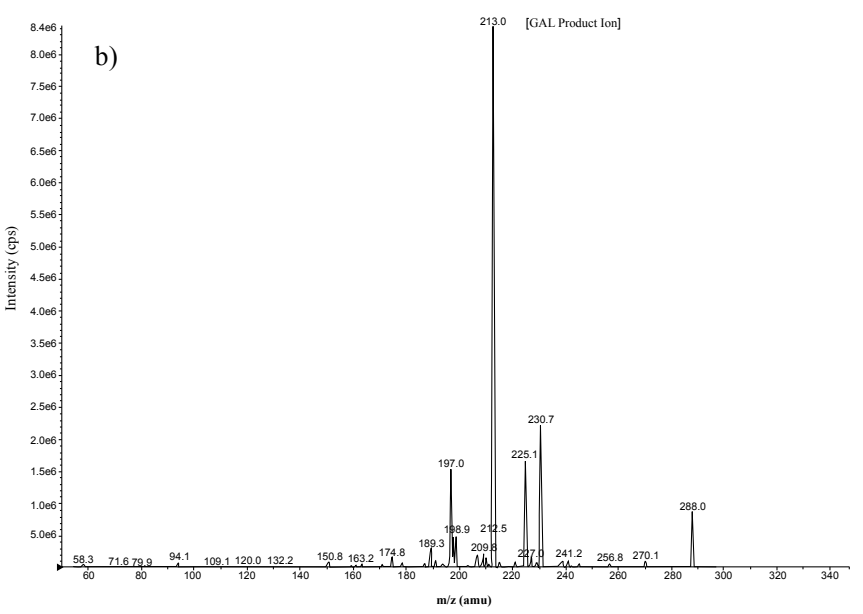

Figure 3: Positive ion mode quantifier precursor to product ion $(\mathrm{M}+\mathrm{H})^{+}$transition of $288-$ to- $213 \mathrm{~m} / \mathrm{z}$ for $\mathrm{GAL}$ is shown as a) full scan $\mathrm{Q} 1$ showing the $\mathrm{GAL}$ precursor $288 \mathrm{~m} / \mathrm{z}$ ion and b) full scan Q3 showing the GAL product $213 \mathrm{~m} / \mathrm{z}$ ion. 



Figure 4: Positive ion mode quantifier precursor to product ion $(\mathrm{M}+\mathrm{H})^{+}$transition of $256-$ to- $167 \mathrm{~m} / \mathrm{z}$ for $\mathrm{DPH}$ is shown as a) full scan Q1 showing the DPH precursor $256 \mathrm{~m} / \mathrm{z}$ ion and b)fullscan Q3 showing the DPH product $167 \mathrm{~m} / \mathrm{z}$ ion.

ion transitions for GAL and DPH were not present the qualifier ion transitions could be used for the quantitation of GAL. The modes of fragmentation of the $288 \mathrm{~m} / \mathrm{z}$ GAL and $256 \mathrm{~m} / \mathrm{z}$ DPH precursor ions producing product ions at an $\mathrm{m} / \mathrm{z}$ of $197,209,213,225,231,270$ for GAL and 152, 167, 183, 230, for DPH have been established in literature $[31,32,34,35]$.

\section{Conclusions}

A new high throughput sample preparation extraction LC/MS/ MS analysis assay has been developed with commercially available materials to enable future researchers the ability to reproducibly and sensitively quantitate galanthamine in guinea pig plasma. The sample preparation employing a $10 \mathrm{~min}$ trichloromethane liquidliquid extraction gave consistent extraction recoveries of GAL and the internal standard DPH from $160 \mu \mathrm{L}$ plasma sample volumes. The LC/MS/MS analysis method operated in the positive ion MRM Turbo Ionspray mode yielding highly selective quantifier precursor to product ion $(\mathrm{M}+\mathrm{H})+$ transitions for GAL (288-to-213 m/z) and DPH (256-to$167 \mathrm{~m} / \mathrm{z}$ ). Sample run times where on the order of 1.50 min per sample. Most importantly, it is envisioned that this new extraction assay with low sample volume requirements, rapid GAL extraction recovery, and rapid sample runtimes would be beneficial to not only a wide range of researchers researching pharmacokinetics, bioavailability, or bioequivalence studies but of more specific interest to researchers exploring applications of organophosphorus nerve agent poisoning countermeasures in guinea pigs and humans.

\section{Acknowledgements}

The authors would like to acknowledge: Dr. James Bruce's Laboratory from the University of Washington Genome Sciences, Seattle, WA; The University of Washington's Proteomics Resource (UWPR95794); Dr. Jeff Jones's Laboratory from Washington State University, Pullman, WA for open use of a variety of liquid chromatography and mass spectrometry instrumentation; and the United States Army Medical Research Institute of Chemical Defense, Aberdeen Proving Grounds, MD, for their continued scientific correspondence and support.

\section{References}

1. Ohbu S, Yamashina A, Takasu N, Yamaguchi T, Murai T, et al. (1997) Sarin poisoning on Tokyo subway. South Med J 90: 587-593.

2. Romano JA Jr, King JM (2001) Psychological casualties resulting from chemical and biological weapons. Mil Med 166: 21-22.

3. Sogorb MA, Vilanova E, Carrera V (2004) Future applications of phosphotries- terases in the prophylaxis and treatment of organophosporus insecticide and nerve agent poisonings. Toxicol Lett 151: 219-233.

4. GROB D (1956) The manifestations and treatment of poisoning due to nerve gas and other organic phosphate anticholinesterase compounds. AMA Arch Intern Med 98: 221-239.

5. Compton JAF (1987) Military Chemical and Biological Agents: Chemical and Toxicological Properties. The Telford Press, Caldwell, New Jersey.

6. Ballantyne B, Marrs TC (1992) Pharmacology and toxicology of organophosphates. Butterworth-Heinemann Oxford 3: 35-39.

7. Williams PT, Hilmas CJ (2010) Cholinergic Effects On Ocular Flutter In Guinea Pigs Following Nerve Agent Exposure: A Review. J Med CBR Def 8: 1-13.

8. Shih TM, Duniho SM, McDonough JH (2003) Control of nerve agent-induced seizures is critical for neuroprotection and survival. Toxicol Appl Pharmacol 188: $69-80$.

9. Bajgar J (2004) Organophosphates/nerve agent poisoning: mechanism of action, diagnosis, prophylaxis, and treatment. Adv Clin Chem 38: 151-216.

10. Newmark J (2007) Nerve agents. Neurologist 13: 20-32.

11. Berman HA, Decker MM (1986) Kinetic, equilibrium, and spectroscopic studies on dealkylation ("aging") of alkyl organophosphonyl acetylcholinesterase. Electrostatic control of enzyme topography. J Biol Chem 261: 10646-10652.

12. Jones DE, Carter WH Jr, Carchman RA (1985) Assessing pyridostigmine efficacy by response surface modeling. Fundam Appl Toxicol 5: S242-S251.

13. Kassa J (2002) Review of oximes in the antidotal treatment of poisoning by organophosphorus nerve agents. 40: 803-816.

14. Wetherell J, Hall T, Passingham S (2002) Physostigmine and hyoscine improves protection against the lethal and incapacitating effects of nerve agent poisoning in the guinea-pig. Neurotoxicology 23: 341-349.

15. Deshpande SS, Viana GB, Kauffman FC, Rickett DL, Albuquerque EX (1986) Effectiveness of physostigmine as a pretreatment drug for protection of rats from organophosphate poisoning. Fundam Appl Toxicol 6: 566-577.

16. Dawson RM (1994) Review of oximes available for treatment of nerve agent poisoning. J Appl Toxicol 14: 317-331.

17. Buckley NA, Karalliedde L, Dawson A, Senanayake N, Eddleston M (2004) Where is the evidence for treatments used in pesticide poisoning? Is clinical toxicology fiddling while the developing world burns? J Toxicol Clin Toxicol 42 113-116.

18. Pereira EF, Hilmas C, Santos MD, Alkondon M, Maelicke A, et al. (2002) Unconventional ligands and modulators of nicotinic receptors. J Neurobiol 53 479-500.

19. Arias E, Alés E, Gabilan NH, Cano-Abad MF, Villarroya M, et al. (2004) Galantamine prevents apoptosis induced by beta-amyloid and thapsigargin: 
Citation: Steiner WE, Pikalov IA, Williams PT, English WA, Hilmas CJ (2012) Development of a Novel LC/MS/MS Extraction Assay for Galanthamine in Guinea Pig Plasma and its Application to Nerve Agent Countermeasures. 1: 149. doi:10.4172/scientificreports. 149

involvement of nicotinic acetylcholine receptors. Neuropharmacology 46: 103114

20. Kihara T, Sawada H, Nakamizo T, Kanki R, Yamashita H, et al. (2004) Galantamine modulates nicotinic receptor and blocks Abeta-enhanced glutamate toxicity. Biochem Biophys Res Commun 325: 976-982.

21. Albuquerque EX, Pereira EF, Alkondon M, Rogers SW (2009) Mammalian nicotinic acetylcholine receptors: from structure to function. Physiol Rev 89: 73-120.

22. Albuquerque EX, Pereira EF, Aracava Y, Fawcett WP, Oliveira M, et al. (2006) Effective countermeasure against poisoning by organophosphorus insecticides and nerve agents. Proc Natl Acad Sci U S A 103: 13220-13225.

23. Hilmas CJ, Poole MJ, Finneran K, Clark MG, Williams PT (2009) Galantamine is a novel post-exposure therapeutic against lethal VX challenge. Toxicol Appl Pharmacol 240: 166-173.

24. Aracava Y, Pereira EF, Akkerman M, Adler M, Albuquerque EX (2009) Effectiveness of donepezil, rivastigmine, and (+/-)huperzine A in counteracting the acute toxicity of organophosphorus nerve agents: comparison with galantamine. J Pharmacol Exp Ther 331: 1014-1024.

25. Maxwell DM, Brecht KM, Lenz DE, O’Neill BL (1988) Effect of carboxylesterase inhibition on carbamate protection against soman toxicity. J Pharmacol Exp Ther 246: 986-991.

26. Hilmas CJ, Apland JP, Deshpande SS, Haigh J, Gordon R, et al. (2006) Pyridostigmine bromide pretreatment protects against soman-induced lethality in the guinea pig. USAMRICD Technical Report ICD 06-19.

27. Claessens HA, van Thiel M, Westra P, Soeterboek AM (1983) Highperformance liquid chromatographic determination of galanthamine, a longacting anticholinesterase drug, in serum, urine and bile. J Chromatogr 275: 345-353
28. Tencheva J, Yamboliev I, Zhivkova Z (1987) Reversed-phase liquid chromatography for the determination of galanthamine and its metabolites in human plasma and urine. J Chromatogr 421: 396-400.

29. Zhao Q, lyer GR, Verhaeghe T, Truyen L (2002) Pharmacokinetics and safety of galantamine in subjects with hepatic impairment and healthy volunteers. $J$ Clin Pharmacol 42: 428-436.

30. Patel BN, Sharma N, Sanyal M, Shrivastav PS (2008) Quantitation of donepezi and its active metabolite 6-O-desmethyl donepezil in human plasma by a selective and sensitive liquid chromatography-tandem mass spectrometric method. Anal Chim Acta 629: 145-157.

31. Verhaeghe T, Diels L, de Vries R, De Meulder M, de Jong J (2003) Development and validation of a liquid chromatographic tandem mass spectrometric method for the determination of galantamine in human heparinzed plasma. $J$ Chromatogr B Analyt Technol Biomed Life Sci 789: 337-346.

32. Nirogi RV, Kandikere VN, Mudigonda K, Maurya S (2007) Quantitative determination of galantamine in human plasma by sensitive liquid chromatography-tandem mass spectrometry using loratadine as an internal standard. J Chromatogr Sci 45: 97-103.

33. Snyder LR, Kirkland JJ, Dolan JW (2009) Polarity Index In: Snyder LR, Kirkland JJ, Dolan JW (Ed) Introduction to Modern Liquid Chromatography. (3rdedn), John Wiley \& Sons, Inc., Hoboken, New Jersey.

34. Kumar S, Rurak DW, Riggs KW (1998) Simultaneous determination of diphenhydramine, its $\mathrm{N}$-oxide metabolite and their deuterium-labeled analogues in ovine plasma and urine using liquid chromatography/electrospray tandem mass spectrometry. J Mass Spectrom 33: 1171-1181.

35. Han D, Chen C, Zhang C, Zhang Y, Tang X (2010) Determination of mangiferin in rat plasma by liquid-liquid extraction with UPLC-MS/MS. J Pharm Biomed Anal 51: 260-263. 